\section{POS0612 EXPLORING EQUIVALENCE BETWEEN BIOSIMILAR SB4 AND REFERENCE ETANERCEPT BY ASSESSING EFFECTIVENESS IN RHEUMATOID ARTHRITIS PATIENTS TREATED IN ORDINARY CLINICAL PRACTICE}

G. Haugeberg ${ }^{1,2}$, G. Bakland ${ }^{3}$, E. Rødevand ${ }^{4}$, I. J. Widding Hansen ${ }^{1}$, A. Diamantopoulos ${ }^{5}$, A. H. Pripp ${ }^{5} .{ }^{1}$ Sorlandet Hospital, Division of Rheumatology, Department of Medicine, Kristiansand, Norway; ${ }^{2}$ Norwegian University of Science and Technology, Department of Neuromedicine and Movement Science, Faculty of Medicine and Health Sciences, NTNU, Trondheim, Norway; ${ }^{3}$ University Hospital of Northern Norway, Department of Rheumatology, Tromsø, Norway; ${ }^{4}$ St Olav's Hospital, Department of Rheumatology, Trondheim, Norway; ${ }^{5}$ Martina Hansens Hospital, Oslo Centre of Biostatistics and Epidemiology, Oslo, Norway

Background: Biosimilar drugs follow a tailored approval pathway that usually includes a Phase III comparative efficacy randomized controlled trial with a high internal but low external validity. Therefore, observational studies with high external validity are important to reassure patients and physicians that there are no clinically meaningful differences in effectiveness between a biosimilar and its reference drug. A EULAR Task Force systematic review and others have noted that recent comparative effectiveness studies often do not disclose applied analytical methods in sufficient detail, with many studies not adjusting for confounders nor accounting for attrition or missing data. ${ }^{1,2}$

Objectives: To apply the EULAR Points to Consider for Comparative Effectiveness Research (CER) in an analysis of reference etanercept (ETN) and SB4 biosimilar ETN in patients with rheumatoid arthritis (RA) treated in ordinary clinical practice in Norway.

Methods: ETN-nalive patients with RA starting ETN treatment between January 2010 and July 2018 at five centres in Norway were followed for at least 1 year; the 2 cohorts remained on either ETN or SB4 throughout. The primary outcome was DAS28 at Week 52. This CER has been designed to formally assess equivalence for DAS28, based on the accepted equivalence margin of 0.6. ${ }^{3}$ Conventional regression and propensity score (PS) models have been applied for the primary outcome evaluation of DAS28 at Week 52. Based on clinical knowledge, the confounders adjusted for in the step-wise PS model were age, gender, DAS28, order of biologics, and concomitant conventional synthetic disease-modifying anti-rheumatic drugs. A standardized difference (d) of $<0.1$ indicates a good match.

Results: In the unmatched sample, there were 575 patients treated with reference ETN and 299 treated with SB4. Before PS matching, baseline mean (SD) DAS28 was different between the ETN and SB4 groups, 4.3 (1.2) vs 4.0 (1.3), (d) $=0.25$. After PS matching, there were 176 RA patients in each group; baseline mean (SD) DAS28 was 4.1 (1.2) vs 4.1 (1.3), (d) = 0.05. At Week 52, the difference (mean [95\% confidence interval $(\mathrm{Cl})]$ ) between reference ETN and SB4 for primary outcome DAS28 at Week 52 was $-0.02(-0.33$ to 0.29$)$ in the unmatched analysis. Since the entire $95 \% \mathrm{Cl}$ is within the pre-defined equivalence margin of 0.6 , equivalence at Week 52 has formally been shown. The analysis of the PS matched groups to Week 52 is ongoing and results will be presented in the poster.

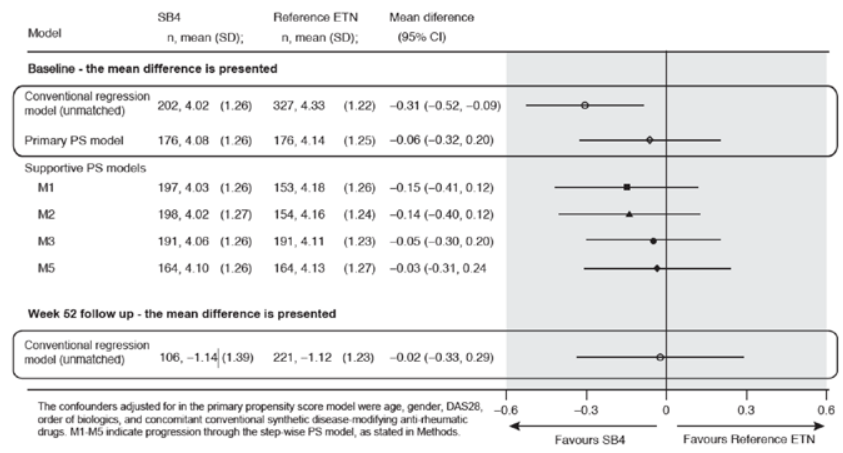

Conclusion: Our results show the importance of adopting proper analytical techniques when comparing a biosimilar with its reference product. A conventional regression model may not fully account for differences in key clinical measures (in this instance, disease activity) between the two groups at baseline, and therefore the Week 52 results might be biased. The propensity score matched model ensures comparability of the groups at baseline and therefore the validity of the Week 52 results should be more robust.

REFERENCES:

[1] Cantini F and Benucci M. Mandatory, cost-driven switching from originator etanercept to its biosimilar SB4: possible fallout on non-medical switching. Ann Rheum Dis 2020; 79: e13.

[2] Lauper K KJ, De Wit M, Fautrel B, et al. A Systematic Review to Inform the EULRA Points to Consider When Analysing and Reporting Comparative
Effectiveness Research With Observational Data in Rheumatology. Annals of the Rheumatic Diseases 2020; 79 .

[3] Fransen $\mathrm{J}$ and van Riel PL. The Disease Activity Score and the EULAR response criteria. Clin Exp Rheumatol 2005; 23: S93-99.

Acknowledgements: The authors wish to acknowledge Janet Addison and Ulrich Freudensprung of Biogen for their intellectual contributions to this abstract and Bjørg Tilde Fevang for providing data from Haukeland University Hospital in Bergen. Editorial support for the preparation of this abstract was provided by Excel Scientific Solutions (Fairfield, CT, USA); funding was provided by Biogen International $\mathrm{GmbH}$.

Disclosure of Interests: Glenn Haugeberg Grant/research support from: Biogen, Gunnstein Bakland: None declared, Erik Rødevand: None declared, Inger Johanne Widding Hansen: None declared, Andreas Diamantopoulos: None declared, Are Hugo Pripp: None declared

DOI: 10.1136/annrheumdis-2021-eular.1604

\section{POS0613 TOCILIZUMAB DECREASES ANGIOGENESIS IN RHEUMATOID ARTHRITIS THROUGH ITS REGULATORY EFFECT ON EMMPRIN/CD147}

D. Zisman ${ }^{1,2}$, M. Safieh ${ }^{1,3}$, E. Simanovich ${ }^{3}$, J. Feld ${ }^{1,2}$, A. Kinarty ${ }^{3}$, L. Zisman ${ }^{3}$, T. Gazitt ${ }^{1}$, A. Haddad ${ }^{1}$, M. Elias ${ }^{1}$, I. Rosner ${ }^{2,4}$, L. Kaly ${ }^{4}$, M. Amit Rahat ${ }^{2,3}$. ${ }^{1}$ Carmel Medical Center, Rheumatology Unit, Haifa, Israel; ${ }^{2}$ Technion-Israel Institute of Technology, Ruth and Bruce Rappaport Faculty of Medicine, Haifa, Israel; ${ }^{3}$ Carmel Medical Center, Immunotherapy Laboratory, Haifa, Israel; ${ }^{4}$ Bnei Zion Medical Center, Rheumatology Unit, Haifa, Israel

Background: Angiogenesis is an important contributor to the development of Rheumatoid arthritis (RA). Tocilizumab (TCZ), an anti-IL-6 receptor antibody, is an immunosuppressant used in the treatment of RA patients, but its effects on angiogenesis and the molecular mechanisms regulating new blood vessel formation are not fully elucidated.

Objectives: To evaluate the concentrations of pro- and anti-angiogenic factors in serum samples of RA patients, before and after the initiation of TCZ treatment and to explore in an in vitro co-culture system the mechanisms of TCZ action. Methods: We evaluated the concentrations of EMMPRIN, VEGF, MMP-9, IL-6 NGAL, endostatin and thrombospondin-1 (Tsp-1) using commercial ELISA kits from 40 RA patients, before and 4 months after the initiation of TCZ treatment The levels of secreted EMMPRIN, VEGF MMP-9 and Tsp- 1 were measured in an in vitro co-culture system of HT1080 fibroblasts and U937 monocytes with and without addition of anti-EMMPRIN blocking antibody. In the tube formation assay serum samples and supernatnats from the co-cultures were added to endothelial layer. Images were obtained after 6 hours of incubation and the number of closed lumens were counted in two separate fields. In the wound assay, supernatants from the co-cultures, with or without the addition of the anti-EMMPRIN antibody were added to the endothelial layer after scratching. The scratch site area was measured immediately and compared to the area after 24 hours of incubation to assess the distance of cell migration.

Results: Study population included 40 RA patients, $33(82.5 \%)$ females, mean age of $57.5 \pm 11.1$ years, disease duration of $7.7 \pm 5.6$ years, and $53.9 \%$ positive for rheumatoid factor initiating treatment with TCZ. In this patient cohort, 25/40 $(62.5 \%)$ patients were classified as "responders" according to EULAR criteria. Following 4 mounts of treatment, statistically significant reductions in the levels of EMMPRIN/CD147 $(p=0.035)$, without significant changes in serum levels of MMP-9, VEGF, MMP-3 and MMP-7 and of the anti-angiogenetic factors Tsp-1 and endostatin were found. A statistically significant decrease in the ratio between the pro-angiogenic factor EMMPRIN and the anti-angiogenic factor Tsp-1 that was calculated for each patient 4 months after initiating TCZ was found $(p=0.031)$. The decrease in angiogenesis was manifested by the reduced number of closed lumen tube-like structures formed by EaHy926 endothelial cell line after incubation with serum samples 4 months after initiation of TCZ, relative to the number of closed lumens formed prior to TCZ initiation $(p=0.007)$. The ratio between EMMPRIN and Tsp-1 was significantly reduced in the responding patients versus non-responders ( $p=0.033$ ), while the levels of VEGF, MMP-9, Tsp-1, and EMMPRIN were unchanged.

In vitro, the accumulation of the pro-angiogenic factors EMMRPIN, VEGF and MMP-9 in the supernatants was increased in the co-culture, while the accumulation of the anti-angiogenic factor Tsp-1 was decreased. When EMMPRIN was neutralized with a blocking antibody, supernatants derived from these co-cultures exhibited reduced migration, proliferation, and tube-like structure formation in functional assays.

Conclusion: Our findings suggest an important role for EMMPRIN in mediating pro-angiogenic signals in RA patients, with EMMPRIN/Tsp-1 ratio serving as a marker of angiogenesis in RA. When administered to RA patients, TCZ in turn, exerts an anti-angiogenic effect through its regulation of EMMRPIN/ CD147 levels.

Disclosure of Interests: None declared

DOI: 10.1136/annrheumdis-2021-eular.1616 